\title{
TITLE:
}

\section{Light emission from silicon in photonic crystal nanocavity}

\author{
$\operatorname{AUTHOR}(\mathrm{S})$ :
}

Fujita, Masayuki; Tanaka, Yoshinori; Noda, Susumu

\section{CITATION:}

Fujita, Masayuki ... [et al]. Light emission from silicon in photonic crystal nanocavity. IEEE JOURNAL OF SELECTED TOPICS IN QUANTUM ELECTRONICS 2008, 14(4): 1090-1097

\section{ISSUE DATE:}

2008-07

URL:

http://hdl.handle.net/2433/84557

\section{RIGHT:}

(c) 2008 IEEE. Personal use of this material is permitted. However, permission to reprint/republish this material for advertising or promotional purposes or for creating new collective works for resale or redistribution to servers or lists, or to reuse any copyrighted component of this work in other works must be obtained from the IEEE. 


\title{
Light Emission From Silicon in Photonic Crystal Nanocavity
}

\author{
Masayuki Fujita, Member, IEEE, Yoshinori Tanaka, and Susumu Noda, Fellow, IEEE
}

\begin{abstract}
We have introduced a photonic crystal into a singlecrystal silicon slab in order to manipulate the light emission. When the lattice constant of a defect-free photonic crystal matches the wavelength of light in the medium, the light emitted from the silicon is resonantly extracted at the photonic band edge within the escape light cone. When the lattice constant is larger than the wavelength, Brillouin zone folding of the photonic band also allows the light to be extracted; we achieved an intensity that was enhanced by a factor of $\sim 20$ due to the diffraction of internal light into the light cone. We have also created a point defect in photonic crystals with smaller lattice constants that functions as a nanocavity and strongly interacts with the silicon emitter. Four cavity modes were observed, with different $Q$-factors and emission patterns. The mode orders were assigned using the resonant wavelengths and polarizations. The observed emission at room temperature was enhanced by a factor of $\sim 30$ in comparison to that of an unprocessed area of siliconon-insulator. Our study demonstrates that employing a photonic crystal nanocavity in silicon can greatly improve the light extraction efficiency, the characteristics of the radiation pattern, and the internal quantum efficiency.
\end{abstract}

Index Terms-Light emission, light extraction, photonic crystal, photonic nanocavity, silicon.

\section{INTRODUCTION}

$\mathbf{S}$ ILICON ( $\mathrm{Si}$ ) is the most pervasive semiconductor material currently used in electronics. Recent progress in the field of Si photonics, which has the potential to overcome many of the limitations of electronics, including the speed of signal processing, has been remarkable [1]-[7]. However, due to the indirect optical transition in $\mathrm{Si}$, efficient Si-based light emitters have not yet been developed. Photonic crystals (PCs), in which the refractive index varies periodically on a length scale comparable to the optical wavelength of interest, have recently attracted much attention as fundamental components of next-generation photonic devices [8], [9]. PCs allow light to be manipulated by controlling the optical modes, which can be achieved by careful design of both the dispersion curves and photon density of states. For example, the light emitted from a PC can be extracted using the Brillouin zone folding of the photonic band [10]-[12]. Light emission can be inhibited by the photonic

Manuscript received December 17, 2007; revised February 3, 2008. This work was supported in part by the Ministry of Education, Culture, Sports, Science, and Technology of Japan through Research Programs for Scientific Research under Grant-in-Aid, COE, and Special Coordination Fund, and in part by the Core Research for Evolutional Science and Technology of the Japan Science and Technology Agency.

The authors are with the Department of Electronic Science and Engineering, Photonic and Electronics Science and Engineering Center, Kyoto University, Kyoto 615-8510, Japan (e-mail: fujita@qoe.kuee.kyoto-u.ac.jp; ytanaka@qoe.kuee.kyoto-u.ac.jp; snoda@kuee.kyoto-u.ac.jp).

Color versions of one or more of the figures in this paper are available online at http://ieeexplore.ieee.org.

Digital Object Identifier 10.1109/JSTQE.2008.918941 bandgap (PBG) [9], [13], [14], which forbids the existence of photonic modes for certain directions of propagation, and enhanced by modifying the spontaneous emission [9], [15]-[19] via the Purcell effect [20]. Exploiting these effects has the potential to improve the efficiency of light emitters. The development of Si-based PC devices is progressing rapidly [21]-[26]. The advantages of using $\mathrm{Si}$ include its high refractive index, the existence of well-developed microfabrication techniques used in electronics, and the availability of both high-quality crystals with low parasitic loss and silicon-on-insulator (SOI) substrates. However, most of the devices reported thus far have been either passive [21], [22], [25] or refractive index-absorption modulation systems [23], [24], [26]. There are very few reports of PC light emitters fabricated from crystalline $\mathrm{Si}$ itself [27], despite the publication of studies of light emission in PCs based on $\mathrm{Si}$, including erbium-doped Si [28], [29], germanium-Si quantum dots [30], [31], and nanocrystalline Si [32]-[34]. In this paper, we systematically investigate the effect of PC structures on the light emission properties of single-crystal $\mathrm{Si}$, which thus far has remained unclear. In Section II, we study the emission of light from an Si PC with no designed defects. We then describe in Section III the emission characteristics of Si photonic nanocavities [9], [21], formed by the introduction of an artificial point defect that disturbs the structural periodicity of the PC. Finally, the conclusion is provided in Section IV.

\section{Defect-Free Photonic Crystal Slabs}

We investigate the optical emission properties of one of the most popular PC structures, the 2-D PC slab consisting of a triangular lattice of air holes shown in Fig. 1. Strong optical confinement in the vertical direction can be achieved, despite the absence of PC periodicity, by using a freestanding Si slab with low-refractive-index air cladding. This results in total internal reflection (TIR) at the slab-air boundaries, where there is a large contrast in refractive indices. As a result, the light inside the slab should be strongly affected by the periodic structure. For example, when the wavelength of light in the medium $\left(\lambda_{\mathrm{m}}\right)$ matches the lattice constant of the PC $(a)$, the light can be diffracted in the vertical direction [35], [36]. When $a$ is much larger than $\lambda_{\mathrm{m}}$, light can still be extracted from the slab by higher-order diffraction via the Brillouin zone folding of the photonic band [10]-[12]. These PC characteristics are scaled by the normalized frequency $a / \lambda$, where $\lambda$ is the wavelength in vacuum. Thus, the PC effect can be systematically studied using PCs with a range of lattice constants.

The experimental samples were fabricated from an SOI substrate, which was made using the SmartCut method [37]. The thickness and resistivity of the $p$-type Si slab (SOI layer) situated 


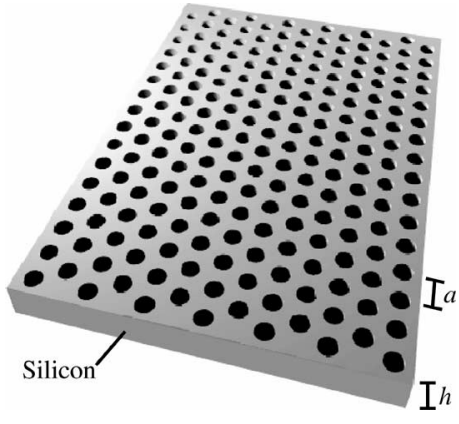

Fig. 1. Schematic picture of 2-D PC slab: $a$ and $h$ denote PC lattice constant and thickness of slab, respectively.
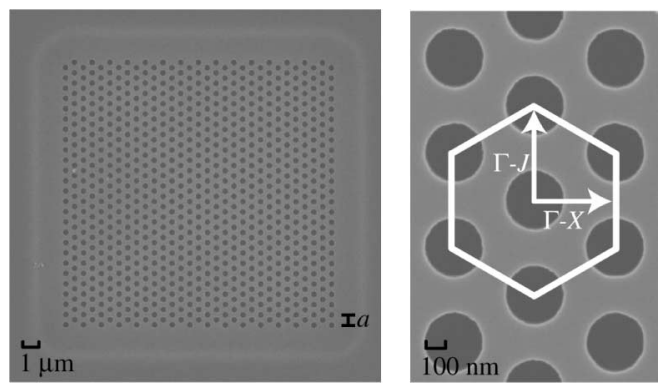

Fig. 2. SEM image of entire fabricated 2-D PC slab (left) and magnified section (right); $a$ denotes the PC lattice constant. $\Gamma-J$ and $\Gamma-X$ represent characteristic directions of the triangular 2-D PC lattice along which photons propagate.

above the $\mathrm{SiO}_{2}$ were $200 \mathrm{~nm}$ and $10 \Omega \cdot \mathrm{cm}$, respectively. The PC patterns were drawn by electron beam lithography using a positive-type resist, ZEON ZEP-520A, and transferred to the $\mathrm{Si}$ slab by $\mathrm{SF}_{6}$-based inductively coupled plasma etching. Finally, the freestanding slab was formed by selective chemical etching of the $\mathrm{SiO}_{2}$ layer on the $\mathrm{Si}$ substrate using an $\mathrm{HF}$ solution. Fig. 2 shows a scanning electron micrograph (SEM) view of the fabricated sample, in which the triangular lattice of air holes is evenly formed. We used 30 PC periods in this study, a number that electromagnetic-field simulations using 3-D finite-difference time-domain (FDTD) method have shown to be sufficient in order to observe the PC effect [38]. The emission properties of the samples were measured using a microphotoluminescence (micro-PL) system at room temperature. Pump light from a continuous-wave (CW) $406 \mathrm{~nm}$ laser was focused on the sample through a numerical aperture (NA) 0.4 objective lens. The emitted light was collected by the same objective lens and measured using a multichannel GaInAs detector system equipped with a monochromator and infrared camera. The irradiated power and spot diameter of the pump light were $10 \mathrm{~mW}$ and $\sim 4 \mu \mathrm{m}$, respectively.

Fig. 3 shows the emission spectra of samples with PC lattice constants ranging in $10 \mathrm{~nm}$ intervals from $a=450$ to $500 \mathrm{~nm}$, in addition to the spectrum of a non-PC sample (an unprocessed SOI area). Well-defined emission peaks are observed close to $1.1 \mu \mathrm{m}$ for the PC samples, corresponding to the electron band edge of Si. The positions of the peak maxima are almost directly proportional to the lattice constant. This implies that the emis-

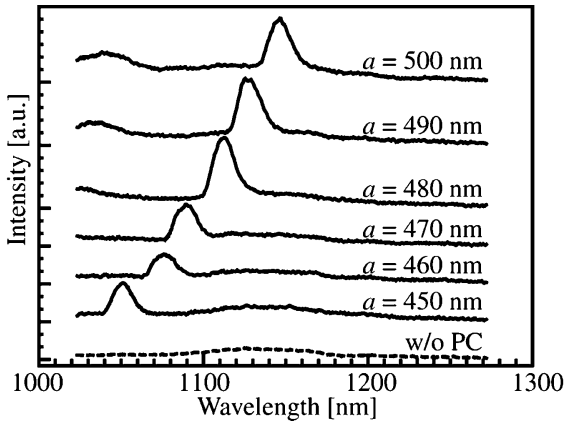

Fig. 3. PL spectra of the 2-D PC slab for various lattice constants $a$. Dashed line denotes the spectrum for an unprocessed SOI area on the same substrate.

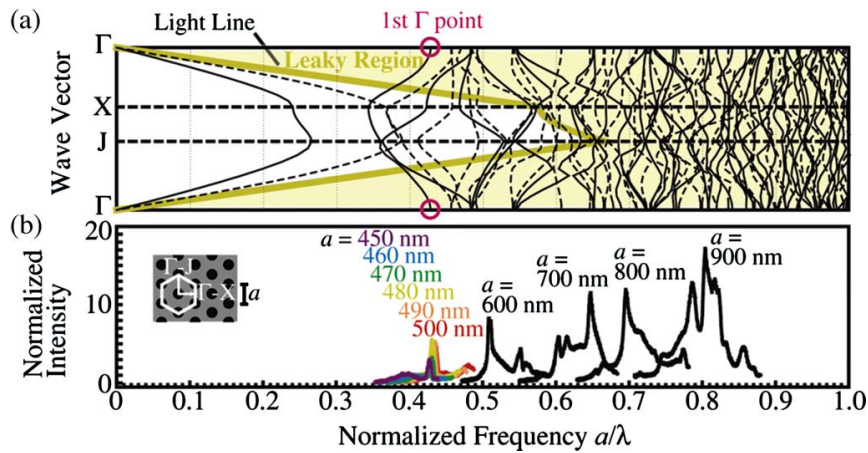

Fig. 4. Spectra for defect-free 2-D PC slabs as a function of normalized frequency. (a) Photonic band diagram calculated using the 3-D plane wave expansion method for $n=3.4, h=0.6 a$, and $r=0.29 a$, where $n$ is the refractive index, $h$ is the slab thickness, and $r$ is the radius of the PC air holes. The solid and dashed curves denote TE and transverse magnetic (TM; the magnetic field is oriented parallel to the slab plane) modes, respectively. Yellow indicates the leaky region where the TIR condition is not satisfied and light is extracted from the slab. The boundary condition for TIR defines the light line. (b) PL intensity versus normalized frequency for different lattice constants. The intensity is normalized by the peak intensity of the unprocessed SOI area in Fig. 3. The inset shows the directions of photon propagation in the PC used in (a).

sion peaks originate from in-plane resonance and that light is extracted at the photonic band edge [35]. In order to investigate the origin of the peaks in more detail, we compare the experimental spectra with the photonic band diagram calculated using the 3-D plane wave expansion method [39]. According to the photonic band structure in Fig. 4(a), the first $\Gamma$-point of the photonic band edge for transverse-electric (TE) polarization (when the electric field is orientated parallel to the slab plane) has a normalized frequency of 0.43 . The spectra shown in Fig. 4(b) for samples with lattice constants between 450 and $500 \mathrm{~nm}$ contain well-defined peaks at this frequency. At this point, the waveguiding modes propagating along the six characteristic in-plane directions inside the slab are coupled to leaky modes in the direction normal to the slab plane at the photonic band edge [35]. The spectra from samples with lattice constants ranging from $600 \mathrm{~nm}$ to $900 \mathrm{~nm}$ at intervals of $100 \mathrm{~nm}$ are also displayed in Fig. 4(b). Emission peaks corresponding to the band edges are again observed for each sample. An increase in the emission intensity across the entire spectrum is found for the PC samples compared to the non-PC sample. This is due to the extraction of light by Brillouin zone folding of the photonic band, which 
diffracts internal light into the escape light cone [12]. The emission intensity is enhanced as the normalized frequency (lattice constant) is increased. This can be explained by the increase of wavenumber components above the light line in Fig. 4(a), where the light is diffracted into the air. For the PC sample with $a=$ $900 \mathrm{~nm}$, the peak emission intensity is 17 times greater than that of the non-PC sample. The enhancement factor is 25 when the reduced emission area due to the air holes is taken into account. This approaches the maximum possible enhancement factor of 40 predicted by FDTD simulation [10]. If nonradiative carrier recombination induced by the $\mathrm{PC}$ fabrication process was significant compared to the intrinsic nonradiative processes of the SOI layer, including Auger recombination [40] and recombination at the SOI surface, no increase of the emission intensity would be observed, due to degradation of the internal quantum efficiency. However, we have observed the PC effect even at room temperature. This indicates that the fabrication of the PC structure has little effect on the internal quantum efficiency of the $\mathrm{Si}$.

\section{Photonic NANOCAVITIES}

One may expect that the introduction of a photonic nanocavity into the PC would lead to even more effective control of the emitted light. Photonic nanocavities allow photons to strongly interact with the PC medium within a tiny space. This would result in an enhancement of the spontaneous emission rate due to the Purcell effect [9], [20]. Nanocavities can also produce distinct radiation patterns for each resonant wavelength. In addition, light at the resonant wavelength of a photonic nanocavity inside a 2-D PC slab can be extracted if there are no additional losses in the cavity, because in-plane emission is inhibited by the PBG effect [9], [41]. We have investigated the properties of the tuned L3 cavity [21], one of the most popular nanocavity structures. This has been used in various applications including add-drop filters [21], [42], nanolasers [43], [44], single-photon sources [18], and the generation of strongly coupled states between a photon and a quantum dot [45], [46]. This type of cavity consists of a 2-D PC slab with a triangular lattice of air holes in which a line of three holes is missing, as shown in Fig. 5. In this study, the holes at each edge of the cavity were shifted outward by $0.1 a$ in order to improve the cavity quality factor, $Q$ [21]. The results presented in Section II, which demonstrate the enhanced emission of light from a 2-D PC Si slab, support our reasoning that a nanocavity might improve the emission characteristics further. It is essential in this system to investigate carefully whether any observed emission peak is due to the cavity mode, because spectral peaks can arise even in the absence of a point defect, as demonstrated in Section II.

We have analyzed the cavity modes for the tuned L3 nanocavity using 3-D FDTD simulations [47], [48]. Fig. 6(a) shows the calculated photonic band diagram and the cavity-mode frequencies for TE polarized light in the slab. Six cavity modes are found in the PBG, for which the localized mode-field intensity distributions are shown in Fig. 6(b). The calculated mode volumes $V_{\mathrm{m}}$ [49] for all six modes are smaller than one cubic wavelength, $(\lambda / n)^{3}$, where $n$ is the refractive index of $\mathrm{Si}$. This indicates the

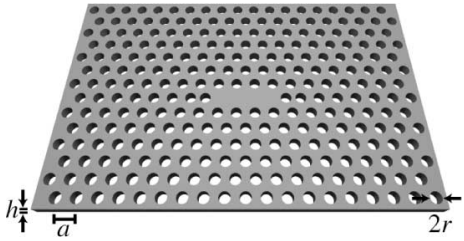

Fig. 5. Schematic picture of the photonic nanocavity. A triangular lattice (lattice constant $a$ ) of air holes (diameter $2 r$ ) forms a 2-D PC in a slab of thickness $h$. The point defect formed by three missing holes acts as a nanocavity.

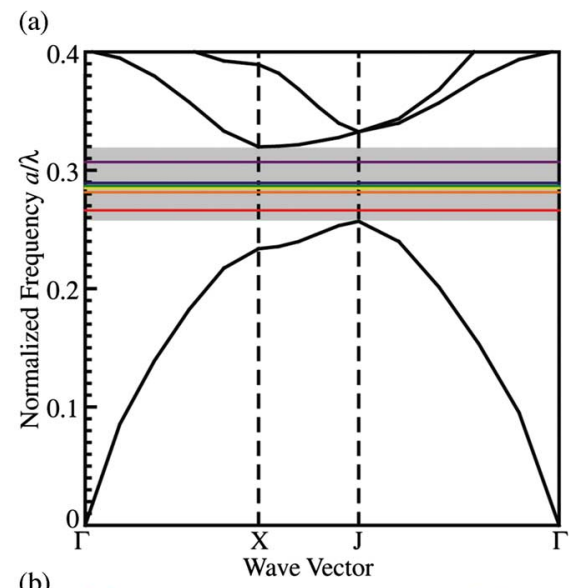

(b)

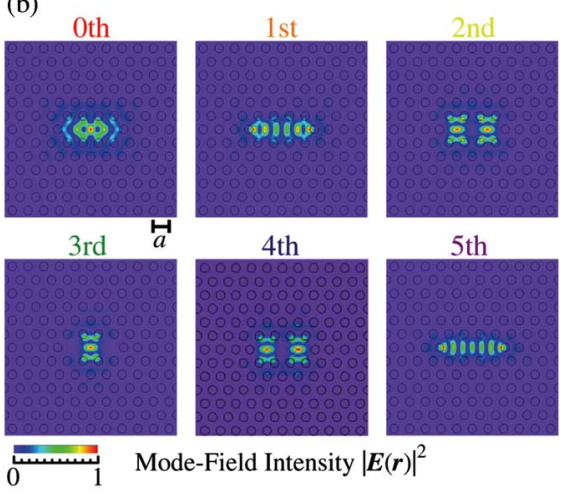

Fig. 6. (a) Photonic band diagram of the 2-D PC slab for the TE mode, calculated using the 3-D FDTD method for $2 r=0.29 a, h=0.6 a, n=3.4$. The shaded gray area represents the PBG region. The horizontal lines indicate the frequencies of the cavity modes. The orders of the cavity modes are labeled 0 th to 5 th in order of increasing frequency. (b) Mode-field (squared electricfield) intensity distribution for each mode order.

presence of a strong interaction between the cavity mode and $\mathrm{Si}$. For conventional applications, only the fundamental (0th) cavity mode with the lowest frequency has attracted much attention [21] because it has the highest $Q$-factor. However, we have studied the properties of various cavity modes in order to investigate how the cavity parameters affect the emission of light from Si.

Samples containing nanocavities with smaller lattice constants than those of the PCs in Section II, ranging from $a=$ 250 to $380 \mathrm{~nm}$ at $10 \mathrm{~nm}$ intervals, were fabricated using the same method. The fabricated samples, an example of which is shown in Fig. 7(a), were investigated at room temperature using the same micro-PL setup, as described in Section II. The wavelength of the CW pump laser was $532 \mathrm{~nm}$, and the irradiated 
(a)

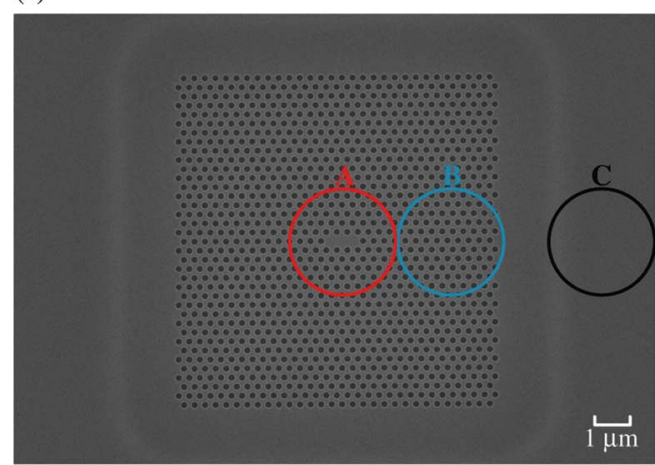

(b)

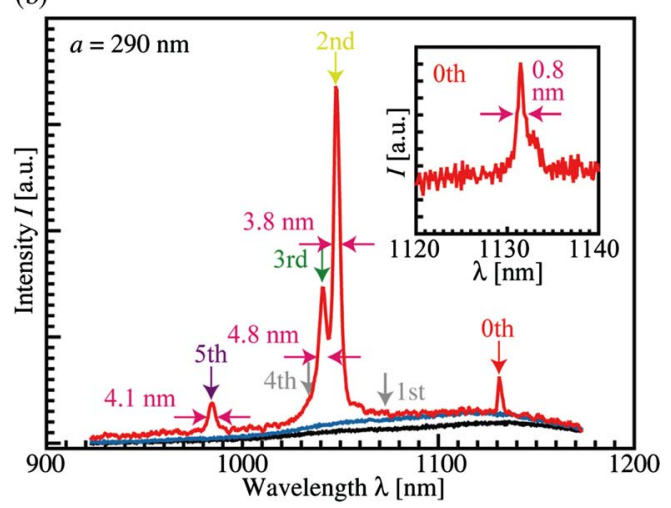

Fig. 7. (a) SEM view of fabricated photonic nanocavity. The circles indicate positions where spectra were measured (A: cavity, B: PC without cavity, C: unprocessed SOI area). (b) PL spectra for each measurement position (sample with $a=290 \mathrm{~nm}$ ). Red, blue, and black spectra were measured at positions A, B, and C, respectively. The mode orders are indicated. Horizontal arrows indicate the linewidths of each mode. The inset shows a high resolution $(0.3 \mathrm{~nm})$ spectrum for the 0 th mode.

power and spot diameter were $6 \mathrm{~mW}$ and $\sim 3 \mu \mathrm{m}$, respectively. Fig. 7(b) shows the PL spectra measured at positions A-C for a sample with $a=290 \mathrm{~nm}$. Well-defined peaks in the band-edge emission of Si close to $1.1 \mu \mathrm{m}$ are absent in the spectrum taken from an unprocessed SOI area (C). Similarly, no well-defined peaks are observed for position $\mathrm{B}$, an area of the PC outside the cavity, despite the similarity of this area to the defect-free PCs investigated in Section II. This is because there are no photonic band edges within the escape light cone that satisfy the leaky condition from the slab for the lattice constant $a=$ $290 \mathrm{~nm}$ (normalized frequency $a / \lambda=0.24-0.32$ ). This lattice constant is smaller than those of the samples that are investigated in Section II and shown in Fig. 4. In contrast, well-defined peaks are observed in the spectrum measured at the cavity position $(A)$. The order of the cavity mode associated with each peak in the spectrum can be assigned using the calculated normalized frequencies in Fig. 6(a). According to Fig. 6, there should be six cavity modes. Fig. 8(a) shows the calculated crosssectional electric field distributions of the nanocavity for the six modes; each mode has a different radiation pattern. Here, the samples were measured using an NA 0.4 objective lens so that only the radiation inside the NA 0.4 light cone could be detected. Fig. 8(b) shows the coupling efficiency to the objective lens $\left(\eta_{\mathrm{c}}\right)$ for each mode, calculated using the 3D FDTD method. The val- (a)

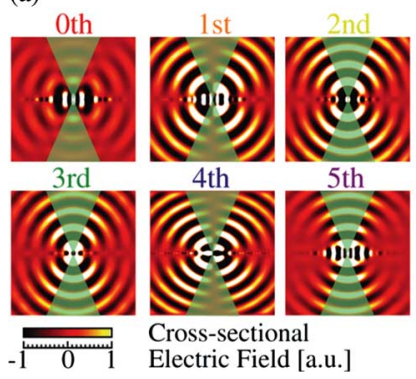

(b)

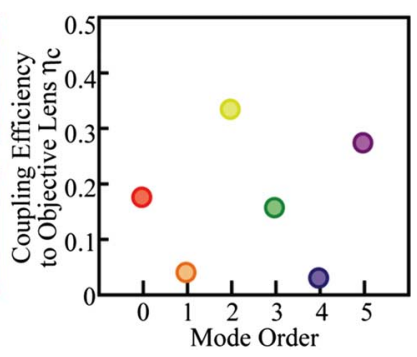

Fig. 8. (a) Calculated cross-sectional electric field distributions of the nanocavity for each mode order. The cavity is at the center of each figure. The green area indicates the NA 0.4 objective lens light cone. (b) Coupling efficiency to NA 0.4 objective lens, $\eta_{\mathrm{c}}$, for each-cavity mode order, calculated using the FDTD method.

ues of $\eta_{\mathrm{c}}$ for the 1st and 4th modes are one order of magnitude smaller than those for the other modes. As a result, the 1st and 4th modes were not visible in the spectra of Fig. 7(b), and only the 0th, 2nd, 3rd, and 5th modes were observed.

In order to validate the origin of each mode in more detail, their individual polarizations were evaluated by measuring spectra with a polarizer placed in front of the detector. The FDTD simulations in Fig. 9(a) show the electric field distributions of each of the four visible modes for both $x$ - and $y$-polarizations. For each mode, one polarization has a symmetric electric field distribution while that of the other polarization is antisymmetric. The polarization originates from the symmetry of the electric field distribution at the slab surface. The total antisymmetric field component becomes zero, but the symmetric component remains. For example, the 0th mode has $y$-polarization because $E_{\mathrm{y}}$ for the 0th mode is symmetric. Fig. 9(b) and (c) shows the polarization-resolved micro-PL spectra from the cavity of the sample measured in Fig. 7(b). The observed polarization directions for each mode are in good agreement with those calculated from the FDTD simulations in Fig. 9(a). These results confirm the validity of the mode assignment in Fig. 7(b).

An important parameter associated with each cavity mode is the $Q$-factor, which is inversely proportional to the rate of loss from the cavity. The experimental $Q$-factors can be estimated using the spectral linewidths and wavelengths of the cavity modes in Fig. 7(b), and are summarized in Fig. 10 for the visible-mode orders. The experimental results are in qualitative agreement with the expectation that the $Q$-factor of the 0th mode is the highest. The theoretical $Q$-factor $\left(Q_{\mathrm{v}}\right)$, which represents optical confinement in the vertical direction, was calculated by the 3-D FDTD method to be 14700 for the 0th mode and 1050 or less for the other modes. The experimental $Q$-factors $\left(Q_{\mathrm{T}}\right)$, which represent the total $Q$-factors, are $1400,280,220$, and 240 for the 0th, 2nd, 3rd, and 5th modes, respectively. The values of $Q_{\mathrm{T}}$ are smaller than theoretically predicted, most likely due to the parasitic optical absorption loss [50], [51] by strong pumping condition with heating [52], [53].

The observed emission intensity $(L)$ for pumping at $\mathrm{CW}$ can be expressed as

$$
L=\eta_{\mathrm{ext}} \times \eta_{\mathrm{c}} \times \eta_{\mathrm{i}} \times V \times J
$$


(a)

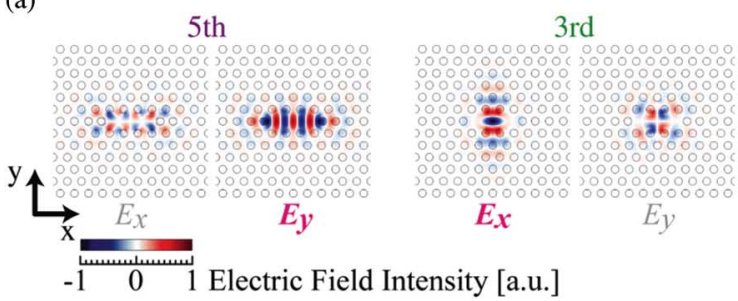

(b)

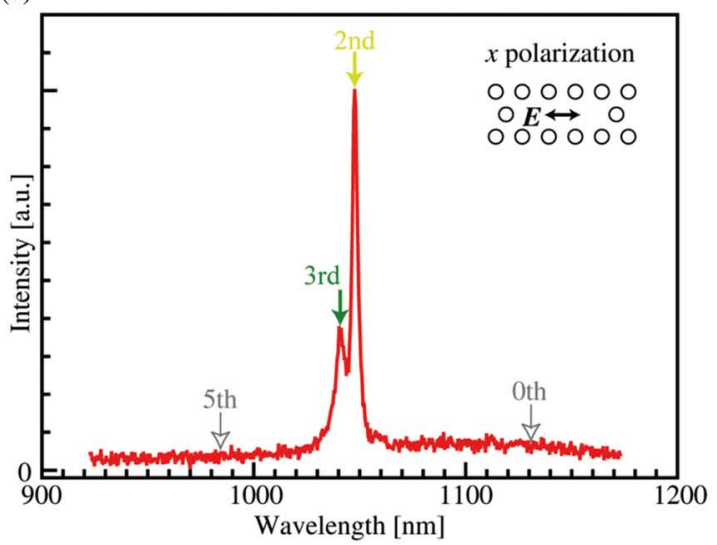

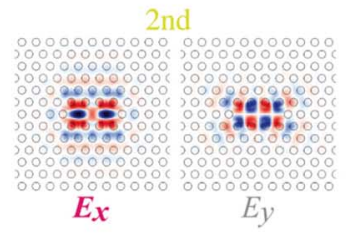

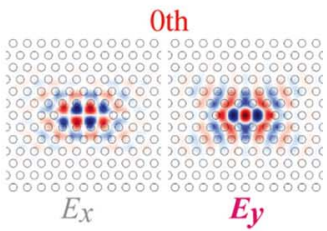

(c)

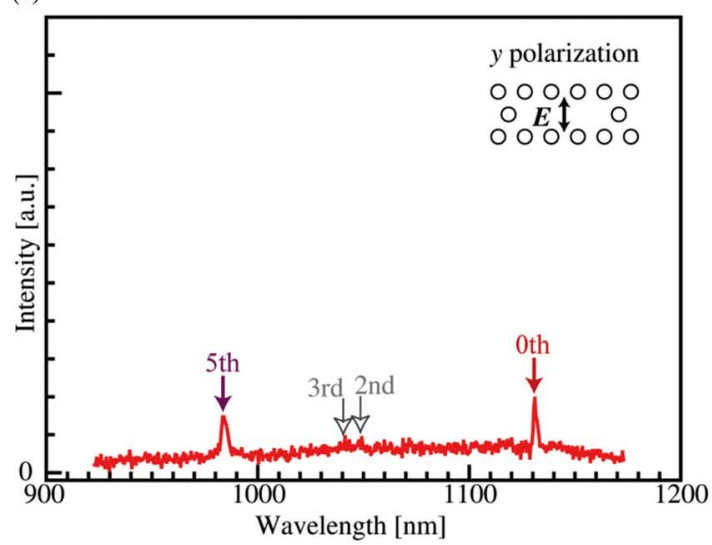

Fig. 9. (a) Electric-field components $E_{x}$ and $E_{y}$ at the slab surface for the visible cavity modes. The field component in red indicates the emitted polarization. (b) Polarization-resolved micro-PL spectra for $x$ and (c) $y$ directions. The cavity-mode orders are indicated.

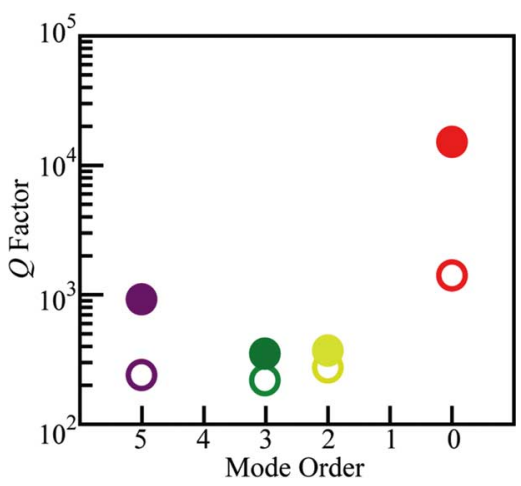

Fig. 10. Cavity $Q$-factors for each mode order. Closed and open circles represent theoretical $Q_{\mathrm{v}}$ calculated by the FDTD method and experimental $Q_{\mathrm{T}}$, respectively.

where $\eta_{\text {ext }}$ is the light extraction efficiency, $\eta_{\mathrm{c}}$ is the efficiency of coupling to the objective lens (shown in Fig. 8), $\eta_{\mathrm{i}}$ is the internal quantum efficiency, $V$ is the emission volume, and $J$ is the pump density. The value of $\eta_{\text {ext }}$ can be calculated from the ratio of the vertical photon leakage to the total optical loss, expressed as $\eta_{\text {ext }}=Q_{\mathrm{T}} /\left(2 Q_{\mathrm{v}}\right)$, where only upward vertical emission is taken into account. Table I shows the estimated light extraction efficiencies for the four-visible mode orders. The extraction efficiency for the 0th mode is only $4.8 \%$ due to a high degree of a parasitic loss and a high value of $Q_{\mathrm{v}}$. The effective emission from the cavity can be estimated by subtracting the spectrum of the region of the PC outside the cavity [position B in Fig. 7(a)] from that at the cavity [position A in Fig. 7]. Fig. 11(a) shows the spectral enhancement due to the cavity, normalized by the spectrum measured from the unprocessed SOI area [position C (a)

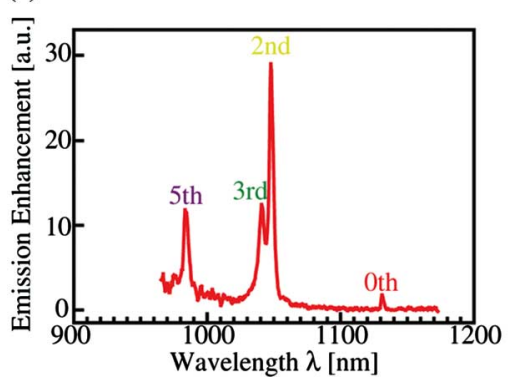

(b)

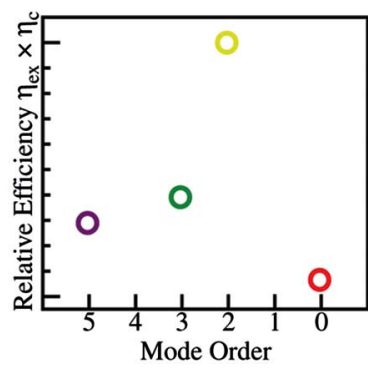

Fig. 11. (a) Enhancement of emission intensity due to the cavity, normalized by the spectrum of the unprocessed SOI area in Fig. 7(b). (b) Product of the light extraction efficiency $\left(\eta_{\text {ext }}\right)$ and the coupling efficiency to the objective $\left(\eta_{\mathrm{c}}\right)$ for each cavity-mode order. Values have been normalized by the maximum for comparison with (a).

in Fig. 7(a)]. The relative spectral enhancement for each mode matches well with the product $\eta_{\mathrm{ext}} \times \eta_{\mathrm{c}}$ of the respective light extraction efficiency and efficiency of coupling to the objective lens, shown in Fig. 11(b). The internal quantum efficiency $\left(\eta_{\mathrm{i}}\right)$ for the cavity can be expressed as

$$
\eta_{\mathrm{i}}=R_{\mathrm{cav}} /\left(R_{\mathrm{cav}}+R_{\mathrm{pc}}+R_{\mathrm{nr}}\right)
$$

where $R_{\text {cav }}$ is the cavity-mode emission rate, $R_{\mathrm{pc}}$ is the emission rate for a defect-free $\mathrm{PC}$, and $R_{\mathrm{nr}}$ is the nonradiative carrier recombination rate. In a nanocavity, the maximum enhancement of $R_{\text {cav }}$ in the ideal case, where the polarization and position of the cavity and emitter are exactly matched, is expected to be a factor of $F_{\mathrm{p}}=\left(3 \lambda^{3} Q_{\mathrm{T}}\right) /\left(4 \pi^{2} n^{3} V_{\mathrm{m}}\right)$ according to the Purcell effect [20]. When comparing the emission efficiencies of the cavity and the original SOI, the difference in emission volumes $V$ 
TABLE I

SUMMARY OF ESTIMATED EFFICIENCIES FOR EACH CAVITY-MODE ORDER

\begin{tabular}{cccccc}
\hline Mode & 0th & 2nd & 3rd & 5th & SOI Wafer \\
\hline \hline$\eta_{\mathrm{ext}}$ & 0.047 & 0.38 & 0.32 & 0.14 & 0.018 \\
$\eta_{\mathrm{c}}$ & 0.17 & 0.33 & 0.15 & 0.27 & 0.15 \\
\hline$L / L_{0}$ & 1.8 & 29 & 13 & 12 & $1\left(=I_{0}\right)$ \\
\hline$\eta_{\mathrm{i}} / \eta_{\mathrm{i} 0}$ & 6.3 & 6.6 & 7.2 & 9.3 & $1\left(=\eta_{\mathrm{i} 0}\right)$ \\
\hdashline$F_{\mathrm{p}}$ & 160 & 28 & 49 & 19 & \\
\hline
\end{tabular}

$\eta_{\mathrm{ext}}$ and $\eta_{\mathrm{c}}$ denote the efficiencies of light extraction and coupling to the objective lens, respectively. The $\eta_{\text {ext }}$ of the unprocessed SOI wafer was calculated using the 3-D FDTD method. The refractive index of $\mathrm{SiO}_{2}$ is assumed to be 1.5. $L / L_{0}$ is the observed enhancement in emission intensity for each cavity mode. $\eta_{\mathrm{i}} / \eta_{\mathrm{i} 0}$ is the estimated increase in the internal quantum efficiency, taking into account the enhancemnet of the emission intensity and the reduction of the emission volume. $F_{\mathrm{p}}$ is the calculated Purcell factor, using the experimental $Q$-factor $Q_{\mathrm{T}}$

(a)

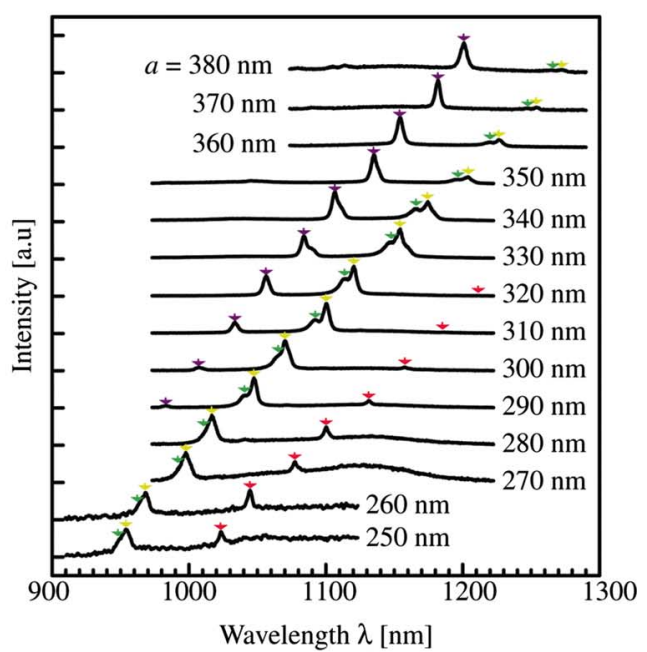

(b)

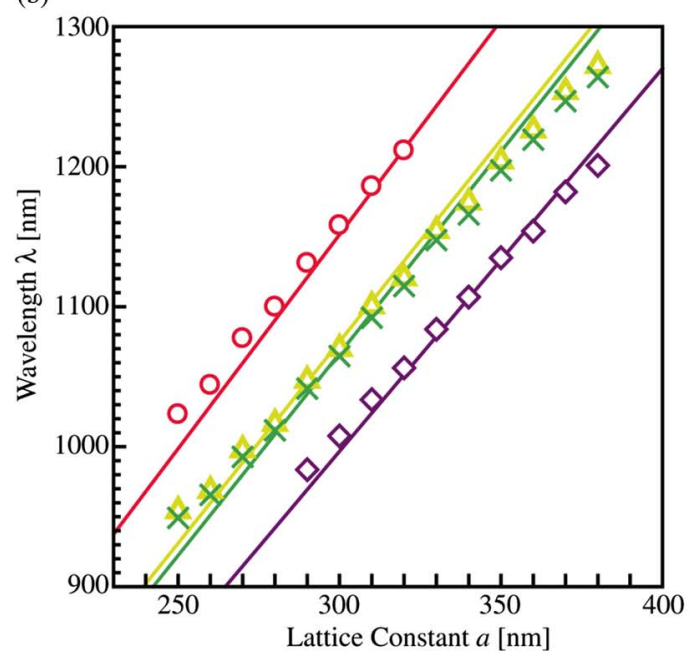

Fig. 12. (a) Emission spectra of PC nanocavities with different lattice constants. Arrows indicate cavity modes. The spectra are normalized by the maximum intensity of each spectrum. (b) Mode wavelength as a function of lattice constant. Red circles, yellow triangles, green crosses, and violet diamonds represent experimental wavelengths for the 0th, 2nd, 3rd, and 5th modes, respectively. Lines indicate wavelengths calculated by the FDTD method. Changes in normalized slab thickness for different lattice constants have been taken into account. should also be taken into account. For the SOI wafer without a PC structure, $V$ can be given as the volume outlined by the pump spot $\left(\pi \times(1.5 \mu \mathrm{m})^{2} \times h=1.4 \mu \mathrm{m}^{3}\right)$. For cavities, $V$ can be approximated as the size of the defect, $4 a \times \sqrt{3} a \times h=$ $0.12 \mu \mathrm{m}^{3}$. Assuming uniform pump density, the increase in the internal quantum efficiency, according to (1), ranges from a factor of 6.3 to 9.3 depending on the mode, as shown in Table I. When $R_{\mathrm{nr}}$ is much larger than the emission rate corresponding to $\mathrm{Si}$, the internal quantum efficiency should be almost proportional to $R_{\text {cav }}$, that is, $F_{\mathrm{p}}$. The fact that these values $\left(\eta_{\mathrm{i}} / \eta_{\mathrm{i} 0}\right)$ are smaller than $F_{\mathrm{p}}$ may be due to the averaged dipole, but they are similar to the spontaneous emission rate enhancements of between 3 and 19 reported for compound semiconductors [15]-[19]. This result strongly suggests that the increase of internal efficiency can occur through the Purcell effect even though the further detailed analysis will be required for the clarification of the carrier distribution.

Finally, we address the effect of varying the lattice constant of PCs containing nanocavities. As shown in Fig. 12, the cavity mode wavelengths become longer as the lattice constant increases. Using the same considerations as for the $a=290 \mathrm{~nm}$ sample in Fig. 11, Fig. 12(a) shows that the enhancement of intensity for the higher order modes is greater than that of the 0th mode for all lattice constants in the measured range. Fig. 12(b) summarizes the observed emission wavelengths as a function of lattice constant. The corresponding values calculated using the FDTD method (solid lines) agree well with the experimental values. This provides additional evidence that the observed spectral peaks indeed correspond to cavity-mode emission from Si photonic nanocavities.

\section{CONCLUSION}

We have successfully demonstrated that PCs and photonic nanocavities can enhance the emission of light from $\mathrm{Si}$, even at room temperature. The extraction of light from Si PCs has been achieved using the photonic band edge, the Brillouin zone folding of the photonic band, or the nanocavity-mode. We have classified the cavity-mode orders of resonance peaks according to their wavelengths and in-plane polarizations. The observed enhancement of emission intensity for each mode can be associated with the light extraction efficiency, which is determined by the cavity $Q$-factor, and the efficiency of coupling to the objective lens. Our experiments suggest that it is possible to improve the internal efficiency in Si even further by controlling spontaneous emission in the nanocavity. To this end, an estimation of the carrier lifetime, a clarification of the nonradiative processes, and the introduction of quantum structures [54] form the next steps toward the further evolution of Si photonics and semiconductor photonic materials, with the eventual goal of realizing $\mathrm{Si}$ lasers.

\section{REFERENCES}

[1] B. Jalali and S. Fathpour, "Silicon photonics," J. Lightw. Techol., vol. 24 no. 12, pp. 4600-4615, 2006.

[2] R. A. Soref, "The past, present, and future of silicon photonics," IEEE J. Sel. Topics Quantum Electron., vol. 12, no. 6, pp. 1678-1687, Nov.-Dec. 2006. 
[3] N. Izhaky, M. T. Morse, S. Koehl, O. Cohen, D. Rubin, A. Barkai, G. Sarid, R. Cohen, and M. J. Paniccia, "Development of CMOS compatible integrated silicon photonics devices," IEEE J. Sel. Topics Quamtum Electron., vol. 12, no. 6, pp. 1688-1698, Nov.-Dec. 2006.

[4] B. Jalali, V. Raghunathan, D. Dimitropoulos, and Ö. Boyraz, "Ramanbased silicon photonics," IEEE J. Sel. Topics Quamtum Electron., vol. 12, no. 3, pp. 412-421, May-June 2006.

[5] L. C. Kimerling, "Silicon microphotonics," Appl. Surf. Sci., vol. 159-160, pp. 8-13, 2000.

[6] B. Jalali, S. Yegnanarayanan, T. Yoon, T. Yoshimoto, I. Rendina, and F. Coppinge, "Advances in silicon-on-insulator optoelectronics," IEEE J. Sel. Topics Quantum Electron., vol. 4, no. 6, pp. 938-947, Nov./Dec. 1998.

[7] R. A. Soref, "Silicon-based group IV heterostructures for optoelectronic applications," J. Vac. Sci. Technol. Vac. Surf. Films A, vol. 14, pp. 913918, 1996.

[8] S. Noda, "Recent progresses and future prospects of two- and threedimensional photonic crystals," J. Lightw. Technol., vol. 24, no. 12, pp. 4554-4567, Dec. 2006.

[9] S. Noda, M. Fujita, and T. Asano, "Spontaneous-emission control by photonic crystals and nanocavities," Nat. Photon., vol. 1, pp. 449-458, 2007.

[10] S. H. Fan, P. R. Villeneuve, J. D. Joannopoulos, and E. F. Schubert, "High extraction efficiency of spontaneous emission from slabs of photonic crystals," Phys. Rev. Lett., vol. 78, pp. 3294-3297, 1997.

[11] M. Boroditsky, R. Vrijen, T. F. Krauss, R. Coccioli, R. Bhat, and E. Yablonovitch, "Spontaneous emission extraction and Purcell enhancement from thin-film 2-D photonic crystals," J. Lightw. Technol., vol. 17, no. 11, pp. 2096-2112, Nov. 1999.

[12] H. Ichikawa and T. Baba, "Efficiency enhancement in surface grating type two-dimensional photonic crystal light emitting diode," Appl. Phys. Lett. vol. 84, pp. 457-459, 2004.

[13] E. Yablonovitch, "Inhibited spontaneous emission in solid-state physics and electronics," Phys. Rev. Lett., vol. 58, pp. 2059-2062, 1987.

[14] M. Fujita, S. Takahashi, Y. Tanaka, T. Asano, and S. Noda, "Simultaneous inhibition and redistribution of spontaneous light emission in photonic crystals," Science, vol. 308, pp. 1296-1298, 2005.

[15] T. Baba, D. Sano, K. Nozaki, K. Inoshita, Y. Kuroki, and F. Koyama, "Observation of fast spontaneous emission decay in GaInAsP photonic crystal point defect nanocavity at room temperature," Appl. Phys. Lett., vol. 85, pp. 3989-3991, 2004.

[16] A. Kress, F. Hofbauer, N. Reinelt, M. Kaniber, H. J. Krenner, R. Meyer, G. Bohm, and J. J. Finley, "Manipulation of the spontaneous emission dynamics of quantum dots in two dimensional photonic crystals," Phys. Rev. B, Condens. Matter, vol. 71, pp. 241304-1-241304-4, 2005.

[17] D. Englund, D. Fattal, E. Waks, G. Solomon, B. Zhang, T. Nakaoka, Y. Arakawa, Y. Yamamoto, and J. Vučković, "Controlling the spontaneous emission rate of single quantum dots in a two-dimensional photonic crystal," Phys. Rev. Lett., vol. 95, pp. 013904-1-013904-11, 2005.

[18] W. H. Chang, W. Y. Chen, H. S. Chang, T. P. Hsieh, J. I. Chyi, and T. M. Hsu, "Efficient single-photon sources based on low-density quantum dots in photonic crystal nanocavities," Phys. Rev. Lett., vol. 96, pp. 117401-1117401-4, 2006

[19] D. G. Gevaux, A. J. Bennett, R. M. Stevenson, A. J. Shields, P. Atkinson, J. Griffiths, D. Anderson, G. A. C. Jones, and D. A. Ritchie, "Enhancement and suppression of spontaneous emission by temperature tuning InAs quantum dots to photonic crystal cavities," Appl. Phys. Lett., vol. 88, pp. 131101-11-31101-3, 2006.

[20] E. M. Purcell, "Spontaneous emission probabilities at radio frequencies," Phys. Rev., vol. 69, p. 681, 1946.

[21] Y. Akahane, T. Asano, B. S. Song, and S. Noda, "High-Q photonic nanocavity in a two-dimensional photonic crystal," Nature, vol. 425, pp. 944-947, 2003.

[22] B. S. Song, S. Noda, T. Asano, and Y. Akahane, "Ultra-high-Q photonic double-heterostructure nanocavity," Nat. Matter., vol. 4, pp. 207-210, 2005.

[23] T. Tanabe, M. Notomi, S. Mitsugi, A. Shinya, and E. Kuramochi, "Fast bistable all-optical switch and memory on a silicon photonic crystal onchip," Opt. Lett., vol. 30, pp. 2575-2577, 2005.

[24] Y. A. Vlasov, M. O'Boyle, H. F. Hamann, and S. J. McNab, "Active control of slow light on a chip with photonic crystal waveguides," Nature, vol. 438, pp. 65-69, 2005.

[25] T. Matsumoto, K. Eom, and T. Baba, "Focusing of light by negative refraction in photonic crystal slab superlens on SOI substrate," Opt. Lett., vol. 31, pp. 2776-2778, 2006.
[26] Y. Tanaka, J. Upham, T. Nagashima, T. Sugiya, T. Asano, and S. Noda, "Dynamic control of the Q factor in a photonic crystal nanocavity," Nat. Matter., vol. 6, pp. 862-865, 2007.

[27] B. Cluzel, V. Calvo, T. Charvolin, E. Picard, P. Noe, and E. Hadji, "Singlemode room-temperature emission with a silicon rod lattice," Appl. Phys. Lett., vol. 89, pp. 201111-1-201111-3, 2006.

[28] M. J. A. de Dood, A. Polman, and J. G. Fleming, "Modified spontaneous emission from erbium-doped photonic layer-by-layer crystals," Phys. Rev. B, Condens. Matter, vol. 67, pp. 115106-1-115106-5, 2003.

[29] Y. S. Choi, J. Y. Sung, S. H. Kim, J. H. Shin, and Y. H. Lee, "Active siliconbased two-dimensional slab photonic crystal structures based on erbiumdoped hydrogenated amorphous silicon alloyed with carbon," Appl. Phys. Lett., vol. 83, pp. 3239-3241, 2003.

[30] M. El Kurdi, S. David, P. Boucard, C. Kammerer, X. Li, V. Le Thanh, and S. Sauvage, "Strong 1.3-1.5 $\mu \mathrm{m}$ luminescence from Ge/Si self-assembled islands in highly confining microcavities on silicon on insulator," J. Appl. Phys., vol. 96, pp. 997-1000, 2004.

[31] J. S. Xia, Y. Ikegami, Y. Shiraki, N. Usami, and Y. Nakata, "Strong resonant luminescence from Ge quantum dots in photonic crystal microcavity at room temperature," Appl. Phys. Lett., vol. 89, pp. 201102-1-201102-3, 2006.

[32] J. Valenta, J. Linnros, R. Juhasz, J. L. Rehspringer, F. Huber, C. Hirlimann, S. Cheylan, and R. G. Elliman, "Photonic band-gap effects on photoluminescence of silicon nanocrystals embedded in artificial opals," J. Appl. Phys., vol. 93, pp. 4471-4474, 2003.

[33] C. D. Presti, A. Irrera, G. Franzò, I. Crupi, F. Priolo, F. Iacona, G. Di Stefano, A. Piana, D. Sanfilippo, and P. G. Fallica, "Photonic-crystal siliconnanocluster light-emitting device," Appl. Phys. Lett., vol. 88, pp. 0335011-033501-3, 2006.

[34] M. Makarova, J. Vučković, H. Sanda, and Y. Nishi, "Silicon-based photonic crystal nanocavity light emitters," Appl. Phys. Lett., vol. 89, pp. 221101-1-221101-3, 2006

[35] M. Imada, A. Chutinan, S. Noda, and M. Mochizuki, "Multidirectionally distributed feedback photonic crystal lasers," Phys. Rev. B, Condems. Matter, vol. 65, pp. 195306-1-195306-8, 2002.

[36] M. Fujita, K. Ishihara, T. Ueno, T. Asano, S. Noda, H. Ohata, T. Tsuji, H. Nakada, and N. Shimoji, "Optical and electrical characteristics of organic light-emitting diodes with two-dimensional photonic crystals in organic/electrode layers," Jpn. J. Appl. Phys., vol. 44, pp. 3669-3677, 2005

[37] M. Bruel, "Application of hydrogen ion beams to silicon on insulator material technology," Nucl. Instrum. Methods Phys. Res. B, vol. 108, pp. 313-319, 1996.

[38] M. Fujita, S. Takahashi, T. Asano, Y. Tanaka, K. Kounoike, M. Yamaguchi, J. Nakanishi, W. Stumpf, and S. Noda, "Controlled spontaneousemission phenomena in semiconductor slabs with a two-dimensional photonic bandgap," J. Opt. A, vol. 8, pp. S131-S138, 2006.

[39] M. Qiu, "Band gap effects in asymmetric photonic crystal slabs," Phys. Rev. B, Condemns. Matter., vol. 66, pp. 033103-1-033103-4, 2002.

[40] M. J. Kerr and A. Cuevas, "General parameterization of Auger recombination in crystalline silicon," J. Appl. Phys., vol. 91, pp. 2473-2480, 2002.

[41] K. Kounoike, M. Yamaguchi, M. Fujita, T. Asano, J. Nakanishi, and S. Noda, "Investigation of spontaneous emission from quantum dots embedded in two-dimensional photonic-crystal slab," Electron. Lett., vol. 41, pp. 1402-1403, 2005.

[42] B. S. Song, S. Noda, and T. Asano, "Photonic devices based on in-plane hetero photonic crystals," Science, vol. 300, p. 1537, 2003.

[43] S. Strauf, K. Hennessy, M. T. Rakher, Y. S. Choi, A. Badolato, L. C. Andreani, E. L. Hu, P. M. Petroff, and D. Bouwmeester, "Self-tuned quantum dot gain in photonic crystal lasers," Phys. Rev. Lett., vol. 96, pp. 1274041-127404-4, 2006.

[44] M. Nomura, S. Iwamoto, K. Watanabe, N. Kumagai, Y. Nakata, S. Ishida, and Y. Arakawa, "Room temperature continuous-wave lasing in photonic crystal nanocavity," Opt. Express., vol. 14, pp. 6308-6315, 2006.

[45] T. Yoshie, A. Scherer, J. Hendrickson, G. Khitrova, H. M. Gibbs, G. Rupper, C. Ell, O. B. Shchekin, and D. G. Deppe, "Vacuum Rabi splitting with a single quantum dot in a photonic crystal nanocavity," Nature, vol. 432, pp. 200-203, 2004

[46] K. Hennessy, A. Badolato, M. Winger, D. Gerace, M. Atature, S. Gulde, S. Falt, E. L. Hu, and A. Imamolu, "Quantum nature of a strongly coupled single quantum dot-cavity system," Nature, vol. 445, pp. 896-899, 2007.

[47] M. Yamaguchi, M. Fujita, W. Stumpf, T. Asano, and S. Noda, "Polarization-resolved resonant excitation of 2D photonic-crystal nanocavity with quantum dots and its optical properties," presented at 
the Ext. Abstr. Autumn Meet. Jpn. Soc. Appl. Phys., Kusatsu, Japan, 2006, vol. 67, no. 29, Paper p-ZD-5.

[48] A. R. A. Chalcraft, S. Lam, D. O’Brien, T. F. Krauss, M. Sahin, D. Szymanski, D. Sanvitto, R. Oulton, M. S. Skolnick, A. M. Fox, and D. M. Whittaker, "Mode structure of the L3 photonic crystal cavity," Appl. Phys. Lett., vol. 90, pp. 241117-1-241117-3, 2007.

[49] R. Coccioli, M. Boroditsky, K. W. Kim, Y. R. Samii, and E. Yablonovitch, "Smallest possible electromagnetic mode volume in a dielectric cavity," in IEEE Proc. Optoelectron., Dec. 1998, vol. 145, no. 6, pp. 391-397.

[50] P. J. Timans, "Emissivity of silicon at elevated temperatures," J. Appl. Phys., vol. 74, pp. 6353-6364, 1993.

[51] G. E. Jellison, Jr. and F. A. Modine, "Optical functions of silicon at elevated temperatures," J. Appl. Phys., vol. 76, pp. 3758-3761, 1994.

[52] K. Inoshita and T. Baba, "Fabrication of GaInAsP/InP photonic crystal lasers by ICP etching and control of resonant mode in point and line composite defects," IEEE J. Sel. Topics Quantum Electron., vol. 9, no. 5, pp. 1347-1354, Sep.-Oct. 2003.

[53] T. Asano, W. Kunishi, M. Nakamura, B. S. Song, and S. Noda, "Dynamical wavelength tuning of channel-drop device in two-dimensional photonic crystal slab," Electron. Lett., vol. 41, pp. 37-38, 2005.

[54] S. Saito, D. Hisamoto, H. Shimizu, H. Hamamura, R. Tsuchiya, Y. Matsui, T. Mine, T. Arai, N. Sugii, K. Torii, S. Kimura, and T. Onai, "Electroluminescence from ultra-thin silicon," Jpn. J. Appl. Phys., vol. 45, pp. L679-L682, 2006.

Masayuki Fujita (S'O0-M'02) received the B.E., M.E. and Ph.D. degrees in electrical and computer engineering from Yokohama National University, Yokohama, Japan, in 1997, 1999, and 2002, respectively.

During his Ph.D. work, he achieved the first room-temperature continuouswave operation of microdisk lasers and the lowest threshold current of $40 \mu \mathrm{A}$ in GaInAsP lasers. He also proposed and demonstrated a microgear laser. In 2002, he joined Quantum Optoelectronics Laboratory at Kyoto University as a Post-Doctoral Fellow and started research on photonic crystals, including the spontaneous emission control in a photonic crystal and the light extraction in organic light-emitting diodes. He is currently an Assistant Professor with the Department of Electronic Science and Engineering at Kyoto University. His research interests include the light emission phenomena in photonic nanostructures and nano-fabrication processing.

Dr. Fujita is a member of the Japan Society of Applied Physics (JSAP) and the Laser Society of Japan. From 1999 to 2002 and from 2003 to 2006, he was a Research Fellow of the Japan Society for the Promotion of Science. He is the recipient of various awards, including the Academic Encouragement Award from JSAP (2001), the JSPS Award for the Most Promising Young Scientist (2006) and the Paper Award from the Laser Society of Japan (2007).
Yoshinori Tanaka received the B.S., M.S., and Ph.D. degrees from Kyoto University, Kyoto, Japan, in 2001, 2003, and 2006, respectively, all in electronics.

From 2003 to 2006, he was a Research Fellow with the Japan Society for the Promotion of Science at Kyoto University. From 2006 to 2007, he was a Postdoctoral Fellow at Kyoto University, where he is currently an Assistant Professor with the Center of Excellence for Education and Research on Photonics and Electronics Science and Engineering. His current research interests include the theoretical analyses of 2-D photonic crystals.

Dr. Tanaka is a member of the Japan Society of Applied Physics (JSAP). He was the recipient of the Academic Encouragement Award from the JSAP in 2004.

Susumu Noda (M'92-SM'06-F'08) received the B.S., M.S., and Ph.D. degrees from Kyoto University, Kyoto, Japan, in 1982, 1984, and 1991, respectively, al in electronics, and an honorary degree from Gent University, Gent, Belgium, in 2006.

From 1984 to 1988, he was with the Mitsubishi Electric Corporation, where he was engaged in research on optoelectronic devices including $\mathrm{AlGaAs} / \mathrm{GaAs}$ distributed-feedback (DFB) lasers and multiple-quantum-well DFB lasers. In 1988, he joined Kyoto University. He is currently a Professor with the Department of Electronic Science and Engineering and the Director of the Center of Excellence for Education and Research on Photonics and Electronics Science and Engineering. His research interest covers physics and applications of photonic and quantum nanostructures.

Prof. Noda is a member of the Japan Society of Applied Physics (JSAP), and a member of the Institute of Electronics, Information, Communication Engineers (IEICE) and the Laser Society of Japan. He was the recipient of various awards, including the IBM Science Award (2000), the JSAP Achievement Award on Quantum Electronics (2005), and the Optical Society of America Joseph Fraunhofer Award/Robert M. Burley Prize. From 2003 to 2005, he served as an IEEE Lasers and Electro-Optics Society (LEOS) Distinguished Lecturer. Since 2007, he has been the IEEE/LEOS Kansai Chapter Chairman. 